\title{
Un ciclo de mejora en Derecho Constitucional
}

\author{
Improvement cycle in \\ Constitutional Law
}

Mạ InMACULAdA JIMÉNEZ-CASTELLANOS BALLESTEROS

https://orcid.org/0000-0001-9514-2869

Universidad de Sevilla,

Departamento de Derecho Constitucional

inmjcb@us.es

DOI: http://dx.doi.org/10.12795/9788447231003.035

Pp.: 735-752 


\section{Contexto del ciclo de mejora}

El ciclo de mejora en el aula (CIMA) que se presenta en este capítulo se llevó a cabo en el curso 2020-2021, en la asignatura de Derecho Constitucional I de primer curso de grado en derecho en la facultad de Derecho de la Universidad de Sevilla. Como consecuencia de las medidas anticovid adoptadas por Real Decreto 926/2020 de 25 de octubre, la Universidad de Sevilla tomó la decisión de adoptar el sistema de clases on line. En consecuencia, la puesta en marcha del CIMA se vio condicionada por esta circunstancia. Esta experiencia se puso en marcha durante un periodo de 8 horas, repartidas en dos semanas. Como dato, en el grupo de clase había matriculados 46 estudiantes y era su primer año en la universidad.

\section{Diseño previo del CIMA}

\section{Mapa de contenidos y problemas}

Tanto el sondeo inicial como los problemas principales que se reflejan en el mapa de contenidos quieren hacer reflexionar al alumno sobre la potestad normativa del gobierno para aprobar disposiciones que tienen rango y fuerza de ley. Dentro de estas normas ocupa un lugar destacado el Decreto Ley. Se diseñó el CIMA en torno a seis preguntas principales que se irían planteando en las sesiones para ser trabajadas a través de las ideas de los alumnos: partiendo de lo que ellos creen saber para gradualmente ir introduciendo conceptos e ideas nuevas (Bain, 2007), y terminar con actividades de contraste.

Ciclos de Mejora en el Aula (2020). Experiencias de Innovación Docente de la US Esta obra se distribuye con la licencia Creative Commons 


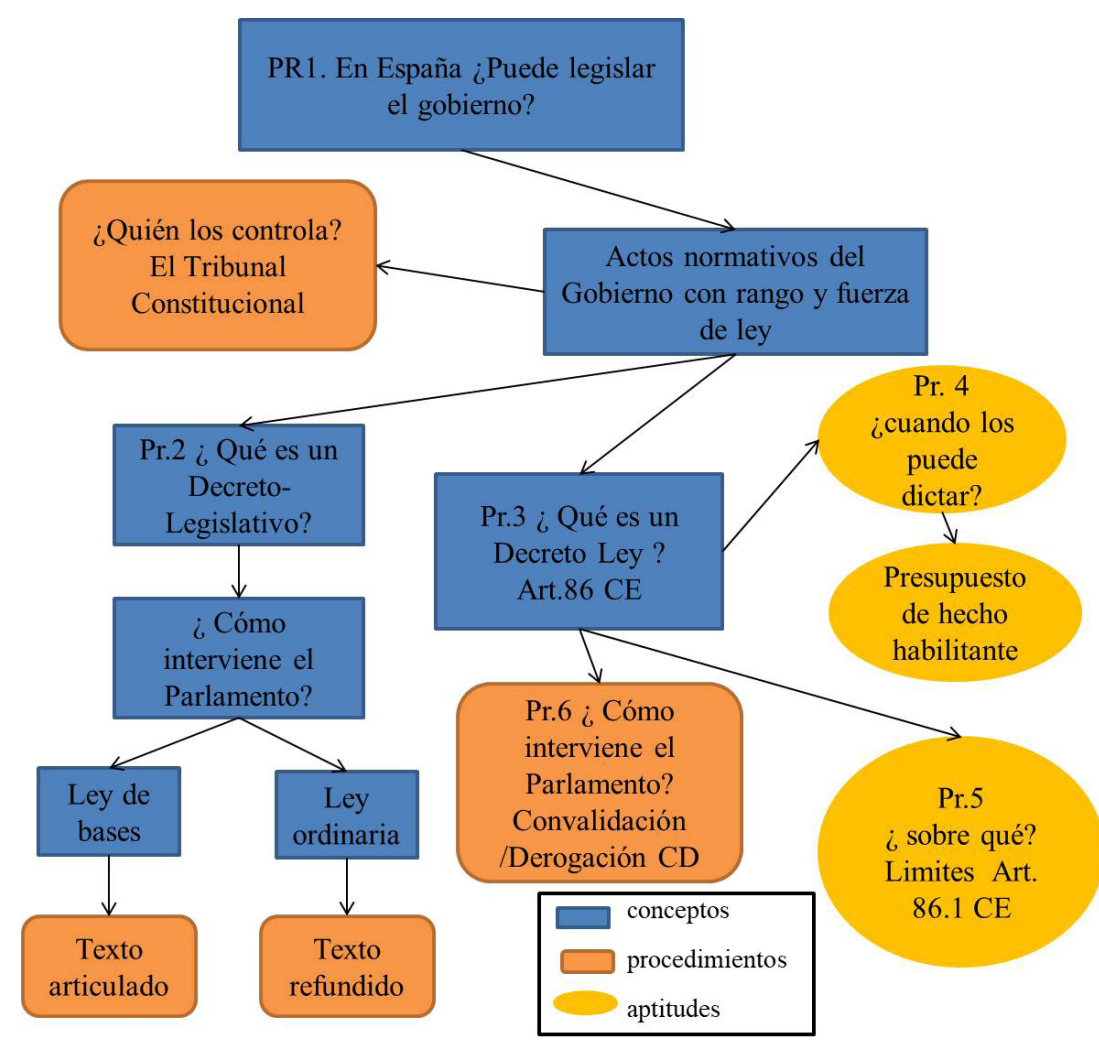

Figura1. Mapa de contenidos y principales preguntas

\title{
Modelo metodológico posible
}

El modelo parte de las ideas previas de los alumnos. A través de preguntas y actividades de contraste se pretende llegar a los contenidos que en realidad queremos que los alumnos aprendan.

\author{
IP IDEAS PREVIAS
}

PR PREGUNTAS

AC ACTIVIDADES DE CONTRASTE

C CONCLUSIÓN

Ciclos de Mejora en el Aula (2020). Experiencias de Innovación Docente de la US Esta obra se distribuye con la licencia Creative Commons Reconocimiento-NoComercial-SinObraDerivada Internacional (CC BY-NC-ND 4.0.) 


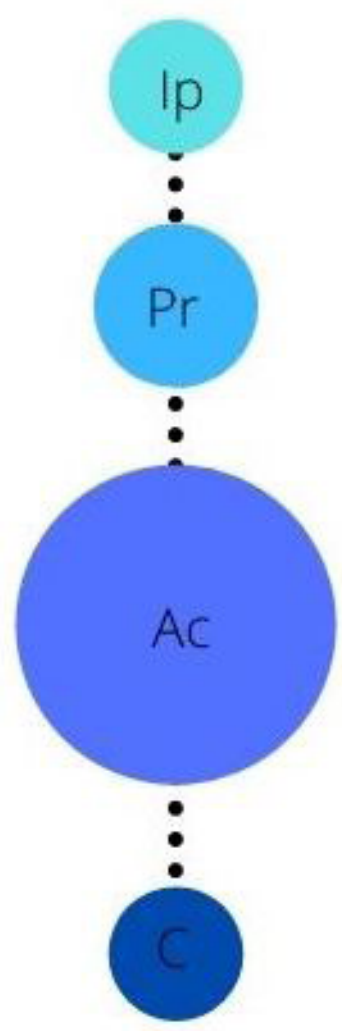

Figura 2. Modelo metodológico posible

\section{Diseño de sesiones}

Se ha seguido el modelo de los talleres conceptuales de Finkel. Los elementos de este taller conceptual serán, en primer lugar, un grupo de alumnos, aproximadamente seis. La pertenencia a un grupo ya genera motivación en los estudiantes. Lo siguiente, una secuencia de preguntas. Sostener un hilo de pensamiento para conseguir que un único proceso de indagación se convierta en una actividad dinámica que incluya varios conceptos específicos. Lo positivo de esta fase, es que pensando juntos es más fácil que el grupo progrese porque, incluso si sus respuestas son incorrectas, lo importante es que están razonando. Al ser

Ciclos de Mejora en el Aula (2020). Experiencias de Innovación Docente de la US Esta obra se distribuye con la licencia Creative Commons 
una secuencia exige una labor por parte de los estudiantes de concentración en aspectos específicos desechando otros accesorios. Los problemas planteados animan a hacer descubrimientos: exigen a los estudiantes que piensen por ellos mismos que saquen conclusiones y luego las apliquen a supuestos nuevos. En definitiva, conducen a un aprendizaje duradero. Por último, el tercer elemento y por ello no menos importante, es un profesor al que acudir. Que esté disponible en la sala cuando los estudiantes estén trabajando. Y ello porque lo estudiantes no se acercarán a los problemas de manera uniforme. Surgirán dudas, errores, preguntas que bajo la dirección del profesor harán la experiencia más fructífera. Para completar este cuadro es necesario conceder un tiempo para que cada grupo exponga los resultados de su investigación y surja el debate entre las diferentes posturas (Finkel, 2000).

Tabla 1. PR1: En España ¿puede legislar el gobierno? Sesión 1 (1hora)

\begin{tabular}{|c|c|c|c|}
\hline 1. & Presentación del problema & PR1 & $15^{\prime}$ \\
\hline \multicolumn{4}{|c|}{$\begin{array}{l}\text { Se divide la clase en } 8 \text { grupos de aproximadamente } 6 \text { alumnos por grupo. Se } \\
\text { les comunica que esta va a ser la forma de trabajar en las próximas sesiones. } \\
\text { Una vez formados, a } 4 \text { de esos grupos se les asigna el rol del gobierno y a } \\
\text { los } 4 \text { restantes el rol de la oposición. Si hay alguien que desee defender } \\
\text { una posición concreta se le permite cambiar de grupo. Deben de defender } \\
\text { argumentos constitucionales a favor y en contra de la pregunta que se les } \\
\text { ha planteado. }\end{array}$} \\
\hline \multicolumn{4}{|c|}{ Recursos: En clase, generación de las Salas en EV } \\
\hline 2. & Debate en grupo & IA & $30^{\prime}$ \\
\hline \multicolumn{4}{|c|}{$\begin{array}{l}\text { El debate se desarrolla dentro de cada grupo. Les comunicaré que deben i } \\
\text { anotando las conclusiones a las que vayan llegando. Tienen que nombra } \\
\text { un portavoz que vaya recogiendo los argumentos, que después se pondrár } \\
\text { en común. La profesora va pasando por cada grupo por si hay dudas } \\
\text { orientándoles donde buscar respuestas a la cuestión planteada. }\end{array}$} \\
\hline \multicolumn{4}{|c|}{ Recursos: Constitución Española } \\
\hline 3. & Exposición de resultados & $A C$ & $15^{\prime}$ \\
\hline
\end{tabular}

Ciclos de Mejora en el Aula (2020). Experiencias de Innovación Docente de la US Esta obra se distribuye con la licencia Creative Commons 
Se hace puesta en común de las conclusiones a las que han llegado los distintos grupos, anotándolas en la pizarra.

Se les invita a ver quién quiere empezar a exponer sus argumentos. Se abre el debate y yo actúo de moderadora. Se procura que todos expongan sus argumentos. Si algún grupo no ha participado a través de su portavoz se les da la palabra. Si alguien da una respuesta que induzca a confusión procedo a aclararla. Una vez expuestas todas las posiciones, se hace una sintesis y se aclaran los conceptos. Y se les vuelve a hacer la misma pregunta. Deben haber llegado a una conclusión afirmativa y se les indica que los instrumentos que usa el gobierno son el Decreto Legislativo y el Decreto Ley.

Recursos: Pizarra

Tabla 2. PR 2: ¿Qué son los decretos legislativos? Sesión 2 (1 hora)

\begin{tabular}{|c|c|c|c|}
\hline 1. & Presentación del problema & PR2 & $10^{\prime}$ \\
\hline \multicolumn{4}{|c|}{$\begin{array}{l}\text { Cuando comiencen esta actividad, los alumnos ya saben que el Gobierno } \\
\text { puede ejercer la potestad legislativa mediante los Decretos legislativos y los } \\
\text { Decretos Leyes, pero no saben nada más. Se les explica que vamos a conocer } \\
\text { algo más sobre el Decreto Legislativo. Para ello vamos a partir de un video. } \\
\text { Luego en grupos y con ayuda de la Constitución Española y de la pág. web el } \\
\text { congreso.es o del parlamento andaluz vamos a contestar a unas preguntas } \\
\text { que pondremos en común a través de otros nuevos portavoces que no sean } \\
\text { los mismos de la vez anterior. }\end{array}$} \\
\hline \multicolumn{4}{|c|}{ Recursos: Ninguno } \\
\hline 2. & ¿Qué es un Decreto Legislativo? & $A C$ & $5^{\prime}$ \\
\hline \multicolumn{4}{|c|}{ Se proyecta en clase } \\
\hline \multicolumn{4}{|c|}{ Recursos: Video } \\
\hline 3. & Debate en grupo & $\mid \mathrm{A}$ & $20^{\prime}$ \\
\hline
\end{tabular}

Ciclos de Mejora en el Aula (2020). Experiencias de Innovación Docente de la US Esta obra se distribuye con la licencia Creative Commons 
Se reparte el mismo cuestionario a cada grupo. Las preguntas son las siguientes:

1. ¿Quién es el autor de un Decreto Legislativo?

2. ¿Qué puede modificar?

3. ¿Qué necesita el gobierno para aprobar un Decreto Legislativo?

4. ¿Para qué sirven?

5. ¿Qué tipos hay?

6. ¿Quién controla estos actos del gobierno?

7. ¿Cuántos Decretos Legislativos se han aprobado en la última legislatura?

8. ¿Dónde se regulan?

Reunidos en grupos deben discutir las respuestas y anotar que argumentos, así como de donde deducen esa respuesta. El alumno portavoz recogerá las respuestas para ponerlas en común. Yo me iré pasando por cada grupo para aclarar dudas.

Recursos: Preguntas de contraste y Constitución Española

\begin{tabular}{|c|c|c|c|}
\hline 4. & Exposición de resultados & AC & $25^{\prime}$ \\
\hline
\end{tabular}

Se hace la puesta en común y se le plantea la primera pregunta al grupo 1. Se abre el debate y se confrontan resultados. Se aclaran dudas y se da una respuesta. Se va pasando a la siguiente pregunta y así sucesivamente para que participen todos los grupos y expongan sus argumentos.

Recursos: Ninguno

Tabla 3. PR 3: ¿Qué son los decretos leyes? Sesión 3 (2 horas)

\begin{tabular}{|l|c|c|c|}
\hline 1 & Presentación de un nuevo problema & Pr.3 & $10^{\prime}$ \\
\hline $\begin{array}{l}\text { Se les recuerda el cuestionario que hicieron en la sesión anterior. Y que } \\
\text { vamos aplicarlo al otro instrumento normativo que tiene el gobierno Decreto } \\
\text { Ley. Se les pide que se reúnan en los grupos y con designen otro portavoz. }\end{array}$ \\
\hline \multicolumn{2}{|l|}{ Recursos: Ninguno } & IA & $20^{\prime}$ \\
\hline 2 & Debate en grupo & \\
\hline
\end{tabular}

Ciclos de Mejora en el Aula (2020). Experiencias de Innovación Docente de la US Esta obra se distribuye con la licencia Creative Commons Reconocimiento-NoComercial-SinObraDerivada Internacional (CC BY-NC-ND 4.0.) 
Se reparte el mismo cuestionario a cada grupo. Las preguntas son las siguientes:

1. ¿Quién es el autor de un Decreto Ley?

2. ¿Qué puede modificar?

3. ¿Que necesita el gobierno para aprobar un Decreto Ley?

4. ¿Para qué sirven?

5. ¿Qué tipos hay?

6. ¿Quién controla estos actos del gobierno?

7. ¿Cuántos Decretos Leyes se han aprobado en la última legislatura?

8. ¿Dónde se regulan?

Deben argumentar constitucionalmente sus respuestas. El alumno portavoz recogerá las respuestas para ponerlas en común. Yo me iré pasando por cada grupo para aclarar dudas.

Recursos: Preguntas de contraste y Constitución Española

\begin{tabular}{|l|l|l|l|}
\hline 3 & Exposición de conclusiones en toda la clase & AC & $30^{\prime}$ \\
\hline
\end{tabular}

Los portavoces ponen en común a toda la clase las conclusiones. Debatimos cada pregunta y su argumentación. Se apuntan en la pizarra. Fijamos conceptos. Y con ello se nos plantea la siguiente pregunta.

Recursos: pizarra

\begin{tabular}{|c|c|c|c|}
\hline 4 & Debate general & AC & $60^{\prime}$ \\
\hline
\end{tabular}

Exponer en base a los dos cuestionarios las diferencias fundamentales entre el decreto ley y decreto legislativo. Se trata de ir volviendo a plantear cada una de las 8 preguntas que resolvieron en los cuestionarios previos e ir viendo las diferencias entre una y otra figura normativa. Algunas diferencias son muy evidentes otras deberán deducirlas de la comparación.

Recursos: Pizarra

Tabla 4. PR.4 ¿Cuándo se puede dictar un decreto ley? Sesión 4 (1 hora)

\begin{tabular}{|l|c|c|c|}
\hline 1 & Presentación del problema & Pr.4 & $10^{\prime}$ \\
\hline \multicolumn{2}{|c|}{$\begin{array}{l}\text { Vamos a tratar sobre qué se entiende por extraordinaria y urgente necesidad. } \\
\text { Se reparte un extracto de una sentencia del Tribunal Constitucional. }\end{array}$} \\
\hline \multicolumn{2}{|l|}{ Recursos: Ninguno } & IA & $30^{\prime}$ \\
\hline 2 & Debate en grupo
\end{tabular}

Ciclos de Mejora en el Aula (2020). Experiencias de Innovación Docente de la US Esta obra se distribuye con la licencia Creative Commons 
Se reparte un extracto de una sentencia del Tribunal. Constitucional. 3 grupos serán la oposición y tendrán que defender los argumentos por los que han impugnado ese Decreto Ley. 3 grupos serán el gobierno. Y los 2 grupos restantes serán el TC

Recursos: Sentencia del Tribunal Constitucional y Constitución Española

\begin{tabular}{|l|l|l|l|}
\hline 3 & Exposición de conclusiones en toda la clase & AC & $20^{\prime}$ \\
\hline
\end{tabular}

Se hace puesta en común de las conclusiones a las que han llegado los distintos grupos, anotándolas en la pizarra.

Se les invita a ver quién quiere empezar a exponer sus argumentos. Se abre el debate y yo actúo de moderadora. Se procura que todos expongan sus argumentos. Si algún grupo no ha participado a través de su portavoz se les da la palabra. Si alguien da una respuesta que induzca a confusión procedo a aclararla. Una vez expuestas todas las posiciones, se hace una síntesis y se aclaran los conceptos. Y se les vuelve a hacer la misma pregunta. Deben haber llegado a una conclusión de que es lo que es un concepto jurídico indeterminado.

Recursos: Pizarra

Tabla 5. Pr.5 ¿Sobre qué materias? Límites de los decretos leyes. Sesión 5 (1 hora)

\begin{tabular}{|c|c|c|c|}
\hline 1 & Presentación del problema & $\operatorname{Pr} .5$ & $10^{\prime}$ \\
\hline \multicolumn{4}{|c|}{$\begin{array}{l}\text { En la sesión anterior se impugnaba el Decreto Ley porque su contenido iba } \\
\text { en contra de la Constitución. }\end{array}$} \\
\hline 2 & Debate en grupo & IA & $30^{\prime}$ \\
\hline \multicolumn{4}{|c|}{$\begin{array}{l}\text { Se reparte un caso práctico con supuestos de Decretos Leyes sobre distintas } \\
\text { materias para que discutan si encajan o no en la Constitución. Se exponen } \\
\text { los argumentos en el aula por grupos. Yo me voy pasando por cada grupo a } \\
\text { orientar y resolver dudas. }\end{array}$} \\
\hline \multicolumn{4}{|c|}{ Recursos: caso práctico y Constitución Española } \\
\hline 3 & Exposición de conclusiones en toda la clase & $A C$ & $20^{\prime}$ \\
\hline \multicolumn{4}{|c|}{$\begin{array}{l}\text { Se hace puesta en común de las conclusiones a las que han llegado los } \\
\text { distintos grupos, anotándolas en la pizarra. Se analiza caso por caso } \\
\text { invitando a los portavoces a que exponga sus argumentos a favor o en contra } \\
\text { y se da una conclusión final. Yo actúo como moderadora y voy aclarando las } \\
\text { dudas que puedan surgir a propósito de los casos que vamos resolviendo. } \\
\text { Al final se hace una síntesis de todo lo que hemos expuesto. }\end{array}$} \\
\hline & izarra & & \\
\hline
\end{tabular}

Ciclos de Mejora en el Aula (2020). Experiencias de Innovación Docente de la US Esta obra se distribuye con la licencia Creative Commons 
Tabla 6. PR 6 ¿Como interviene el Parlamento? (en el decreto ley) Sesión 6 (2 horas)

\begin{tabular}{|c|c|c|c|}
\hline 1 & Presentación del problema & Pr.6 & $10^{\prime}$ \\
\hline \multicolumn{4}{|c|}{$\begin{array}{l}\text { Vamos a tratar de la tramitación parlamentaria de los Decretos Leyes. } \\
\text { ¿Como se realiza? Se les proyectará un video y se les insta buscar la } \\
\text { tramitación parlamentaria que han seguido tres decretos leyes y sus } \\
\text { efectos. }\end{array}$} \\
\hline \multicolumn{4}{|c|}{ Recurso: ninguno } \\
\hline 2 & Convalidación/Derogación & $A C$ & $10^{\prime}$ \\
\hline \multicolumn{4}{|c|}{ Se les proyecta un video sobre el procedimiento } \\
\hline \multicolumn{4}{|c|}{ Recursos: video } \\
\hline 3 & Debate en grupo & IA & $30^{\prime}$ \\
\hline \multicolumn{4}{|c|}{$\begin{array}{l}\text { En grupo deberán buscar en internet la tramitación parlamentaria que han } \\
\text { seguido } 3 \text { decretos leyes. Tres grupos analizarán el convalidado, Tres grupos } \\
\text { el convalidado y tramitado como ley y dos grupos el derogado }\end{array}$} \\
\hline \multicolumn{4}{|c|}{ Recursos: internet y la Constitución Española } \\
\hline 4 & Debate general & $A C$ & $30^{\prime}$ \\
\hline \multicolumn{4}{|c|}{$\begin{array}{l}\text { Se explican en clase la tramitación parlamentaria que han seguido los } 3 \\
\text { decretos leyes. Se anotan en la pizarra los pasos y los efectos en cada caso. } \\
\text { Se establecen las diferencias entre los tres supuestos. }\end{array}$} \\
\hline \multicolumn{4}{|c|}{ Recursos: Pizarra } \\
\hline 5 & Puesta en común & $A C$ & 40' \\
\hline \multicolumn{4}{|c|}{$\begin{array}{l}\text { Se les invita a que hagan una recapitulación de las cuestiones que aún no } \\
\text { hayan quedado claras. }\end{array}$} \\
\hline Recl & & & \\
\hline
\end{tabular}

\section{Cuestionario inicial}

Con carácter previo a la puesta en marcha del CIMA se invitó a los alumnos a que contestaran un cuestionario para conocer el punto del que partíamos. Debian responder de forma anónima.

Ciclos de Mejora en el Aula (2020). Experiencias de Innovación Docente de la US Esta obra se distribuye con la licencia Creative Commons 
El contenido del cuestionario era el siguiente:

SONDEO INICIAL ¿QUé es Un DECRETO LEY?

Con los conocimientos que tienes como ciudadan@, sin consultar Google ni ningún manual, ni la Constitución, ni legislación alguna, me interesa mucho saber tu opinión sincera sobre las cuestiones que vamos a trabajar en los próximos días. Para que te puedas expresar con toda libertad, contesta CON UN NOMBRE INVENTADO lo que piensas sobre los siguientes TRES supuestos de hecho. Procura ser lo más preciso posible, argumenta tus respuestas y di todo lo que piensas. Gracias.

Imagínate que somos un grupo de diputados de la oposición (da igual la orientación política, nos interesan tus argumentos jurídicos) que tenemos que adoptar una posición común razonada acerca del tema de este sondeo

1. Qué opinión te merecería que mañana el gobierno aprobara un decreto-ley que modificara de golpe 14 leyes. Explica en profundidad tus argumentos.

2. Ante las protestas que está planteando el proyecto de ley del gobierno en materia educativa, concretamente que el castellano deje de ser lengua oficial en algunas Comunidades Autónomas, la ministra de educación va a anunciar que esta materia se regulará por Decreto Ley. ¿Qué te parece esta postura del gobierno desde un punto de vista jurídico? Aporta todos los argumentos que apoyen tu punto de vista.

3. Como miembros de la oposición ¿cómo propones que controlemos que el gobierno haga un uso correcto del decreto-ley? Concreta todo lo posible tu propuesta y los argumentos que la apoyan.

El cuestionario fue acogido con entusiasmo y salvo excepciones, casi todos lo enviaron oportunamente. Posteriormente se llevó a cabo la aplicación del CIMA.

Ciclos de Mejora en el Aula (2020). Experiencias de Innovación Docente de la US Esta obra se distribuye con la licencia Creative Commons 


\section{Aplicación del CIMA}

\section{Diario de sesiones: descripción de que ocurrió en clase.}

En la primera sesión se constituyen los grupos on line que habíamos ensayado anteriormente. Algunos tuvieron problemas de conexión, pero fue algo puntual. Antes de incorporarse a cada grupo les recuerdo que tienen que contestar la primera pregunta: en España ¿puede legislar el gobierno? Aparentemente todo el mundo manifiesta plena disposición a trabajar en grupo. Dentro ya de cada espacio de trabajo, algunos se muestran muy activos, otros más tranquilos. En general, mucho más participativos que en ocasiones anteriores y argumentando, utilizando para ello la Constitución Española. Mi sorpresa es enorme pues hasta ese momento no los había visto tan participativos. Ya en la sala principal, cada portavoz nos presenta los argumentos que se han expuesto a favor y en contra. El debate resulta muy interesante y extraemos las principales conclusiones. Al finalizar les pregunto si les ha gustado la clase y me contestan afirmativamente. Alguno comenta que resulta muy dinámico dar las clases así.

Las sesiones siguientes se desarrollaron en la misma línea. Salvo algún problema técnico como que no se escucha el vídeo que les proyecto, todo fue más o menos como lo había planificado en la secuencia de actividades. Se encuentran muy receptivos a la idea de trabajar en grupo y van cambiando los portavoces para que puedan participar todos en el debate general. No obstante, a partir del primer cuestionario comienzan a plantearse dudas sobre las preguntas que necesitan aclaraciones por mi parte. Me voy pasando por los grupos y les recomiendo que las planteen en la sala principal. Estas cuestiones las explico en el debate común, pero es verdad que exigen más detenimiento

Ciclos de Mejora en el Aula (2020). Experiencias de Innovación Docente de la US Esta obra se distribuye con la licencia Creative Commons 
y esto va ralentizar todo el ciclo de mejora. Por tanto, aunque he intentado ceñirme al tiempo, lo cierto es que me ha costado mucho. He preferido fijar conceptos imprescindibles a impartir materias que en mi docencia anterior no hubiera dejado de explicar por nada del mundo.

Entre todas las sesiones destacaría la que les obligaba a comentar un extracto de una sentencia del Tribunal Constitucional. Frente a la pasividad habitual, prácticamente toda la clase se le había leído. Después del debate en grupo, expusieron las ideas principales que habían alegado los recurrentes, el gobierno y el Tribunal Constitucional. A pesar de que algunos repetían las mismas palabras que recogía el texto de la sentencia, sin apenas razonar sobre lo que se les estaba preguntando, fueron muchos los que elaboraron una argumentación propia y con espíritu crítico nos explicaron que habían comprendido acerca de la cuestión que se les planteaba. La puesta en común fue muy enriquecedora. En general, tengo que decir que ha sido una experiencia muy satisfactoria.

\section{Evaluación del aprendizaje de los estudiantes.}

La evaluación se llevó a cabo analizando los sondeos inicial y final que realizaron los alumnos. La conclusión es que hubo mejores resultados en todas las cuestiones que se les fueron planteando. Las escaleras constan de 5 niveles que van desde las respuestas más simples a las más complejas, que coincidían con el nivel de lo que quería que los estudiantes aprendieran. A continuación, se muestran las escaleras de aprendizaje correspondientes a cada una de las preguntas del sondeo.

1. Qué opinión te merecería que mañana el gobierno aprobara un decreto-ley que modificara de golpe 14 leyes. Explica en profundidad tus argumentos. 


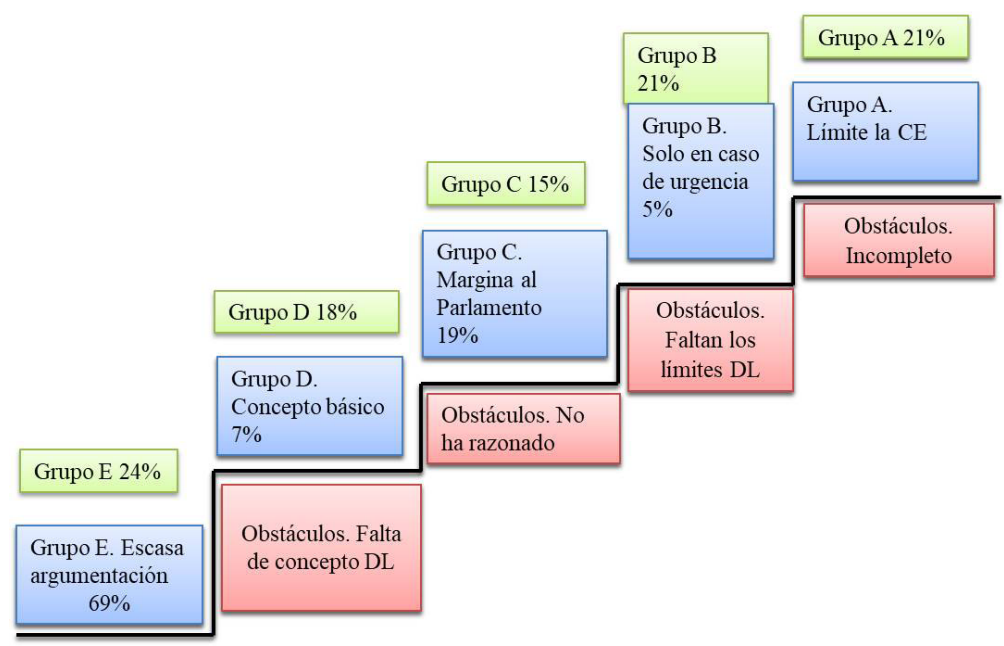

Figura 3. Escaleras de aprendizaje (I)

2. Ante las protestas que está planteando el proyecto de ley del gobierno en materia educativa, concretamente que el castellano deje de ser lengua oficial en algunas Comunidades Autónomas, la ministra de educación va a anunciar que esta materia se regulará por Decreto Ley. ¿Qué te parece esta postura del gobierno desde un punto de vista jurídico? Aporta todos los argumentos que apoyen tu punto de vista.

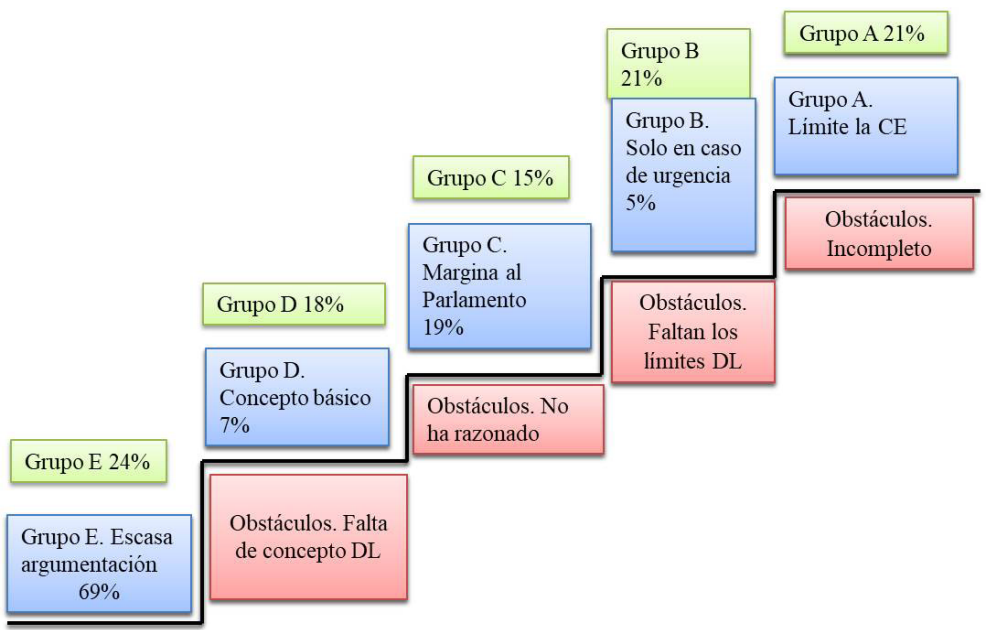

Figura 4. Escaleras de aprendizaje (II)

Ciclos de Mejora en el Aula (2020). Experiencias de Innovación Docente de la US Esta obra se distribuye con la licencia Creative Commons Reconocimiento-NoComercial-SinObraDerivada Internacional (CC BY-NC-ND 4.0.) 
3. Como miembros de la oposición ¿cómo propones que controlemos que el gobierno haga un uso correcto del decreto-ley? Concreta todo lo posible tu propuesta y los argumentos que la apoyan.

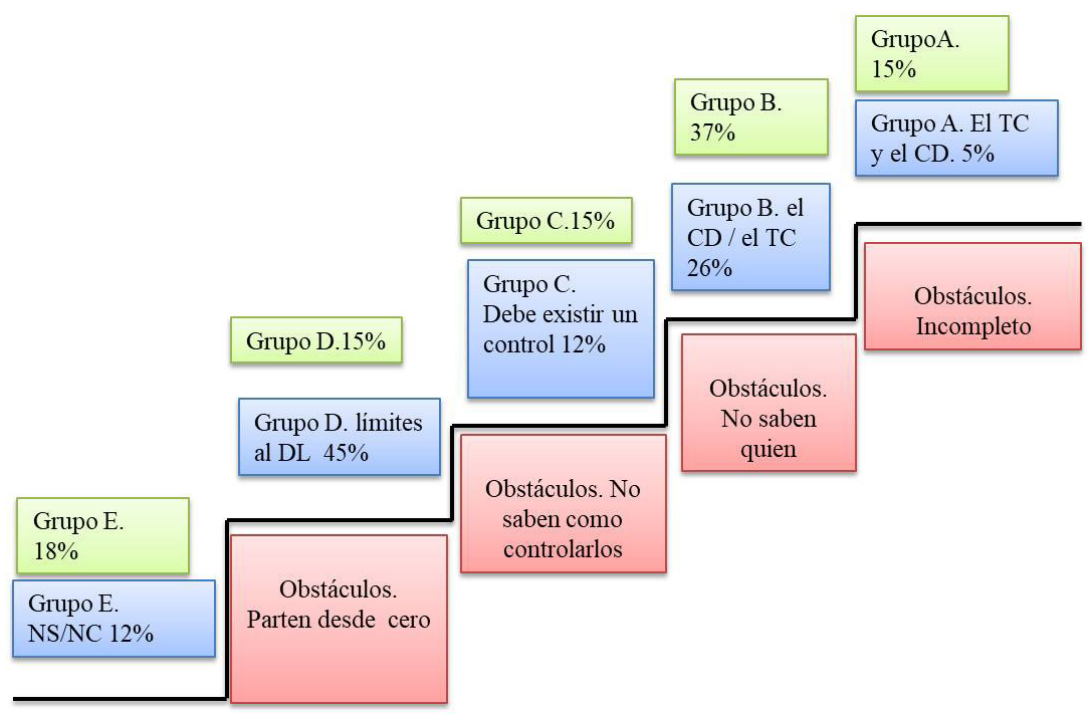

Figura 5. Escaleras de aprendizaje (y III)

\section{Evaluación del CIMA}

\section{Acerca de la valoración final.}

Una vez concluido el CIMA creo que la experiencia ha sido muy satisfactoria. Destacaría de su aplicación el trabajo en grupo, es decir el mayor protagonismo que han ido adquiriendo los estudiantes a lo largo de las sesiones. Asimismo, el carácter dinámico de las clases y su adaptación a las necesidades de los alumnos. Ha resultado mucho más interesante ir introduciendo conocimientos a medida que ellos van haciendo descubrimientos que presentarles toda la materia de una vez.

Ciclos de Mejora en el Aula (2020). Experiencias de Innovación Docente de la US Esta obra se distribuye con la licencia Creative Commons 
La principal dificultad ha sido la falta de tiempo. Las sesiones se han quedado cortas para debatir sobre las cuestiones que se han planteado. Esto es el inconveniente principal con el que me he encontrado.

En cuanto a las mejoras pienso que para un nuevo CIMA se podrían recoger las principales conclusiones, en un resumen, al final de cada clase. Es decir, dividir los power point/temas de cada lección, en partes, y presentarlas a medida que se van tratando los problemas a modo de síntesis. También incluiría otras herramientas tipo wooclap en clase para conocer las ideas previas de las que parten los estudiantes. Por último, introduciría más lectura de resoluciones judiciales acompañadas de cuestionarios para que aprendan a trabajarlos. Y finalmente ajustaría mejor los tiempos de las actividades pues resultan claramente insuficientes.

\section{Principios didácticos.}

En relación con el aprendizaje con los alumnos y de cara al futuro, un principio que he aprendido en el curso y que estoy dispuesta a seguir aplicando es el de indagar en los modelos mentales e ideas de los alumnos (Porlán, 2017). Me ha parecido fundamental como punto de partida y como instrumento para ver la evolución en el aprendizaje. Con anterioridad no tenía en cuenta esto y con frecuencia notaba la falta de motivación en los estudiantes. En segundo lugar, en cuanto a los contenidos de las enseñanzas, estaba acostumbrada a que había que dar todos los contenidos conceptuales, cumplir con el programa sin pensar en lo que quería que mis estudiantes aprendieran. A día de hoy, le doy menos importancia a los contenidos conceptuales y más a las aptitudes. Me ha resultado muy útil aprender a organizar los contenidos sobre la base de problemas clave porque cualquier contenido parte de una pregunta y representarlo en un mapa de contenidos, permite precisamente poner más énfasis en lo que de verdad 
quieres que aprenda el alumno. Por último, en cuanto a la metodología, el principio que quiero que inspire mi modelo didáctico es el de apostar más porque el alumno investigue, busque contenidos, sea más activo y tutorizarlo yo luego en la clase. En definitiva, el principio sería favorecer la participación del estudiante mediante la formación de grupos, los debates y la puesta en común. Asimismo, la necesidad de no improvisar y recurrir al diseño previo de una secuencia de actividades, definiendo los objetivos concretos y planificando todo lo que hacemos en el aula para que los alumnos aprendan ese contenido concreto. Para concluir, en cuanto a la evaluación, he asumido que debo ser realista y no idealizar. He aprendido y me gustaría poner en práctica de aquí en adelante una evaluación secuencial real.

Ciclos de Mejora en el Aula (2020). Experiencias de Innovación Docente de la US Esta obra se distribuye con la licencia Creative Commons 
Palabras clave: Derecho Constitucional, Grado en Derecho, Docencia Universitaria y experimentación docente universitaria

Keywords: Constitutional Law, Law degree, university teaching, university teaching experimentation

\section{Referencias Bibliográficas}

Porlán, R et al (2017). Enseñanza universitaria. Cómo mejorarla. Madrid: Editorial Morata.

Finkel, D (2000). Dar clase con la boca cerrada. Valencia: Publicaciones Universidad de Valencia.

Bain, K (2007). Lo que hacen los mejores profesores de Univerșidad. Valencia: Publicaciones Universidad de Valencia.

Ciclos de Mejora en el Aula (2020). Experiencias de Innovación Docente de la US Esta obra se distribuye con la licencia Creative Commons 